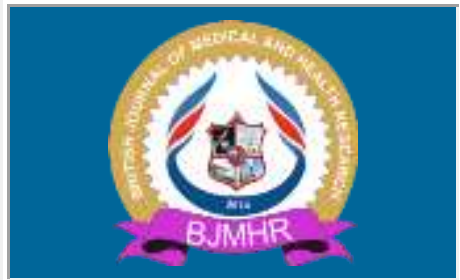

\title{
BJMHR
}

British Journal of Medical and Health Research Journal home page: www.bjmhr.com

\section{Evaluation of Drug-Related Problems among Medical Intensive Care Unit Patients; the Role of Clinical Pharmacist}

\author{
Mina Aghili , N. K. Meera \\ Department of Pharmacy Practice, Visveswarapura Institute of Pharmaceutical Sciences, \\ Rajiv Gandhi University of Health Sciences, Bangalore, India.
}

\section{ABSTRACT}

The complex nature of care provided in the intensive care unit (ICU) usually expose critically ill patients to poly-medication which makes pharmacological treatment as a significant risk factor for the occurrence of drug-related problems (DRPs). The current study aimed to identify and quantify DRPs by the clinical pharmacist among medical ICU (MICU) patients. The prospective observational study was conducted for a period of 6 months (January-June 2019) in 10-beds MICU of a tertiary care academic hospital located in Bangalore, India. For evaluation of DRPs, daily prescribed medications, and overnight emergency medications were analyzed by the pharmacist. Identified DRPs were classified based on Pharmaceutical Care Network Europe. All detected DRPs were reported during the daily medical round to MICU physicians. A total of 153 patients met inclusion criteria and were monitored by the pharmacist. Ninety-nine $(64.7 \%)$ patients were male, and $54(35.3 \%)$ patients were female. The mean age of $51.8 \pm 18.4$ years with an average length of MICU stay $9.3 \pm 3.8$ days were observed. In the current study, overall 240 DRPs were identified. The most common types of DRPs were drugdrug interaction $(69,28.7 \%)$, guideline nonconformity $(53,22.1 \%)$, and drug dose too high $(25,10.4 \%)$. Antibiotics were the most common medication involved in DRPs. Clinical pharmacist received $80.4 \%$ acceptance at the time of interventions. The collaboration of clinical pharmacist with multidisciplinary MICU healthcare team is beneficial for identifying and reporting DRPs among critically ill patients.

Keywords: Drug-related problems, Critically ill patients, Clinical pharmacist. 


\section{INTRODUCTION}

The intensive care units (ICU) are considered as high-risk settings where drug-related problems (DRPs) occur commonly among critically ill patients and may lead to iatrogenic potentially life-threatening consequences [1]. Adult medical intensive care unit (MICU) are at high risk to experience DRPs compared with non-ICU patients, and most of the time patients and their caregivers remain unaware of such errors [2]. DRPs become an additional risk factor for patient morbidity, prolonged length of stay (LOS), and death. There is a complex interplay of many factors for the occurrence of DRPs in critical care settings $[3,4]$. These factors include patientrelated factors (severity of illness found to be the strongest predictor of occurrences of DRPs), medication-related factors (number of medications in ICU is twice as many medications prescribed as for patients in other units which in turn increase the probability of DRPs and medication interactions), ICU complex environment-related factors (high stress, the difficulty of work in this complex environment) and physician-related factors (a lack of pharmacotherapeutic knowledge and skills of prescribers) [5-8]. The clinical pharmacist can contribute to ICU care team by proactive participation in a daily medical round, where a pharmacist can deliver drug information, monitoring the efficacy of pharmacological treatment, prevention, identification and reporting DRPs $[9,10]$. Therefore the current study aimed to identify and quantify DRPs by the clinical pharmacist among MICU patients.

\section{MATERIALS AND METHOD}

The prospective observational study has received the approval from Institutional Human Ethics Committee of Visveswarapura Institute of Pharmaceutical Sciences, Bangalore, India (Reference number: VIPS/IEC/2016-08) and conducted for a period of 6 months from January to June 2019.

\section{Study Site}

MICU (10-beds) at Kempegowda Institute of Medical Science Hospital and Research Center, Bangalore, India.

\section{Inclusion criteria}

Patients ( $\geq 18$ years) admitted to MICU and stayed for a minimum period of 24 hours in MICU. Informed consent has been taken from the patients satisfying these criteria and included in the study.

\section{Exclusion criteria}

Patients admitted to MICU and subsequently discharged on days when the researcher was absent from MICU. Patients who were discharged against medical advice or discharged at request. 


\section{Study Procedure}

Patient-related data including demographic data (age, gender), current medical diagnosis, laboratory data, progress note, medication order, details of medication administration, medical and medication history were recorded in a suitably designed data collection form. Throughout the working hours, clinical pharmacist attended medical rounds, documented newly prescribed medications and carefully reviewed progress note and medication chart for fresh complaints/advice.

All these patient-related data were evaluated for the occurrence of DRPs. Drug-drug interactions (DDIs) were identified by using Lexicomp® drug interaction. Identified DRPs were classified based on Pharmaceutical Care Network Europe (PCNE). DRPs have been brought to the notice of treating physician during the medical round for further action or comment.

\section{Statistical analysis}

Descriptive statistics were used for calculation of mean, standard deviation, frequencies, and percentages of patient's demographics, clinical characteristics, and DRPs. The incidence rate of DRPs was calculated per 1000 patient MICU-days. The statistical package for social sciences for Windows, version 22.0 was used for the analysis of study data.

\section{RESULTS AND DISCUSSION}

A total of 153 patients were included during the study period from January to June 2019. Included patients 'medication charts were reviewed and evaluated by the clinical pharmacist for the occurrence of any DRPs. Demographic analysis of study patients showed that 99 $(64.7 \%)$ patients were male, and $54(35.3 \%)$ patients were female. The mean age of $51.8 \pm 18.4$ years with an average LOS in MICU $9.3 \pm 3.8$ days have been identified. Majority of study patients $(99,64.7 \%)$ were observed to have 1-3 numbers of morbidities. Modes of admission to MICU were found to be direct admission (84, 54.9\%) followed by ICU admission (51, $33.3 \%)$, and normal ward admission $(18,11.8 \%)$ (Table 1). Patients $(18,11.8 \%)$ who required a higher level of care were admitted from normal ward to MICU. These patients were intratransferred from a lower level of care (normal ward) to a higher level of care (MICU) based on the change in their clinical status. Several factors can contribute to intra-transferring of patients from lower to a higher level of care. These factors include system-based factors (e.g., inadequate protocol), human-based factors (e.g., knowledge-based error) rule-based error (e.g., patient assessment inadequate), and skilled-based error (e.g., inexperienced). Moreover, DRPs was found to be one of the prominent incidents identified during intra-transferring of patients to a higher level of care [11]. Also, intra-transferring of patients increase rate of patient mortality and LOS [12]. Overall 240 DRPs were identified in the current study with an 
incidence rate of 91.6 per 1000 patient MICU-days. Types of detected DRPs were classified based on PCNE. The most common types of identified DRPs were an inappropriate combination of drugs (DDIs) $(69,28.7 \%)$, inappropriate drug according to guidelines (guideline nonconformity) $(53,22.1 \%)$, and drug dose too high $(25,10.4 \%)$ (Table 2). Our types of identified DRPs are largely in line with lists of DRPs in other studies $[13,14]$. Our most common types of DRPs indicate a lack of drug knowledge among prescribers which resulted in the occurrence of the majority of the knowledge-based type of DRPs (DDIs, guideline nonconformity). Examples of most common types of DRPs are presented in Table 3. According to Lexicomp® drug interaction, there is major interaction between Sildenafil and Nitroglycerin, such interaction is contraindicated and should be avoided. Sildenafil enhances the vasodilatory effect of Nitroglycerin which can lead to severe hypotension.

Inappropriate selection of antibiotics for infectious diseases such as urinary tract infection, pyelonephritis, pneumonia, acute watery diarrhea, and chronic obstructive pulmonary disease was related to guideline nonconformity type of DRPs. Also, the selection of methylxanthines in the management of acute exacerbation of the chronic obstructive pulmonary disease (COPD) was found to be guideline nonconformity type of DRPs.

There is no evidence supporting the clinical importance of the addition of methylxanthines to bronchodilators and glucocorticoids for the management of acute exacerbation of COPD [15]. In addition to lack of efficacy, methylxanthines can cause adverse effects such as tremor, palpitations, and arrhythmias [16-18]. Analysis of medication involved in identified DRPs showed that antibiotics were found to be the most common class of medications $(32.5 \%$ of the total number of DRPs) involved in DDIs, guideline nonconformity and drug dose too high (Figure 1). The clinical pharmacist made interventions with physicians and received $80.4 \%$ (n $=193$ ) acceptance at the time of interventions. Our high rate of acceptance indicates clinically significant interventions performed by the clinical pharmacist. The beneficial impact of clinical pharmacist in the pharmacotherapeutic evaluation of critically ill patients has been established in hallmark studies $[19,20]$. The beneficial collaboration of clinical pharmacist with healthcare providers include improving the quality, efficiency, safety of medication-related therapies, and removing unnecessary cost burden, especially in reducing DRPs [21-24].

Table 1: Demographic and clinical characteristics of study patients.

\begin{tabular}{lc}
\hline Total number of study patients & $\mathbf{1 5 3}$ \\
\hline Age in years (mean \pm standard deviation) & $51.8 \pm 18.4$ \\
Male & $99(64.7 \%)$ \\
Female & $54(35.3 \%)$ \\
Average number of drugs per prescription (mean \pm standard deviation) & $11 \pm 5.4$ \\
Average length of stay in MICU in days (mean \pm standard deviation) & $9.3 \pm 3.8$ \\
Mode of admission to MICU, from: & $\mathbf{N}(\mathbf{\%})$ \\
Direct admission & $84(54.9)$ \\
\hline \hline
\end{tabular}




\begin{tabular}{lr}
\hline ICU admission & $51(33.3)$ \\
NW admission & $18(11.8)$ \\
Number of morbidities & $\mathbf{N}(\%)$ \\
$1-3$ & $99(64.7)$ \\
$4-6$ & $48(31.4)$ \\
$\geq 7$ & $6(3.9)$ \\
\hline MICU, medical intensive care unit; ICU, intensive care unit; NW, normal ward.
\end{tabular}

Table 2: Types of identified drug-related problems $(\mathrm{N}=\mathbf{2 4 0})$.

\begin{tabular}{lcc}
\hline Type of drug-related problems & N & $(\%)$ \\
\hline Drug selection & & \\
Inappropriate drug according to guidelines (guideline nonconformity) & 53 & 22.1 \\
Inappropriate drug (within guidelines but otherwise contraindicated) & 20 & 8.3 \\
Inappropriate duplication of drug & 8 & 3.3 \\
No drug treatment in spite of existing indication (untreated condition) & 5 & 2.1 \\
Too many drugs prescribed for indication (over treating) & 15 & 6.3 \\
Inappropriate combination of drugs (drug-drug interaction) & 69 & 28.7 \\
Drug form & & \\
Inappropriate drug form & 4 & 1.7 \\
Dose selection & & \\
Drug dose too low & 8 & 3.3 \\
Drug dose too high & 25 & 10.4 \\
Dosage regimen not frequent enough & 4 & 1.7 \\
Dose timing instructions wrong, unclear or missing & 12 & 5 \\
Treatment duration & & \\
Treatment duration too short & 5 & 2.1 \\
Others & & \\
Inadequate drug monitoring & 12 & 5 \\
\hline
\end{tabular}

Table 3: Examples of most common types of drug-related problems.

\begin{tabular}{|c|c|}
\hline $\begin{array}{l}\text { Drug-related } \\
\text { problems }\end{array}$ & Examples \\
\hline \multirow{5}{*}{$\begin{array}{l}\text { Inappropriate } \\
\text { combination of drugs } \\
\text { (drug-drug interaction) } \\
\text { Inappropriate drug } \\
\text { according to guidelines } \\
\text { (guideline } \\
\text { nonconformity) }\end{array}$} & Nitroglycerine-Sildenafil \\
\hline & Tolvaptan-Sodium chloride (3\%) \\
\hline & Clopidogrel-Taurine \\
\hline & $\begin{array}{l}\text { Inappropriate selection of antibiotics for infectious diseases such } \\
\text { as urinary tract infection, pyelonephritis, pneumonia (CAP, HAP, } \\
\text { AP), acute watery diarrhea, and chronic obstructive pulmonary } \\
\text { disease. }\end{array}$ \\
\hline & $\begin{array}{l}\text { Methylxanthines in management of acute exacerbation of the } \\
\text { chronic obstructive pulmonary disease. There is no evidence } \\
\text { supporting the clinical importance of the addition of } \\
\text { methylxanthines to bronchodilators and glucocorticoids for } \\
\text { management of acute exacerbation of chronic obstructive } \\
\text { pulmonary disease. In addition to lack of efficacy, } \\
\text { methylxanthines can cause adverse effects such as tremor, } \\
\text { palpitations, and arrhythmias. }\end{array}$ \\
\hline Drug dose too high & $\begin{array}{l}\text { Piperacillin-tazobactam in patients with renal impairment } \\
\text { Meropenem in patients undergoing hemodialysis }\end{array}$ \\
\hline
\end{tabular}


CAP, community-acquired pneumonia; HAP, hospital-acquired pneumonia; AP, aspiration pneumonia.

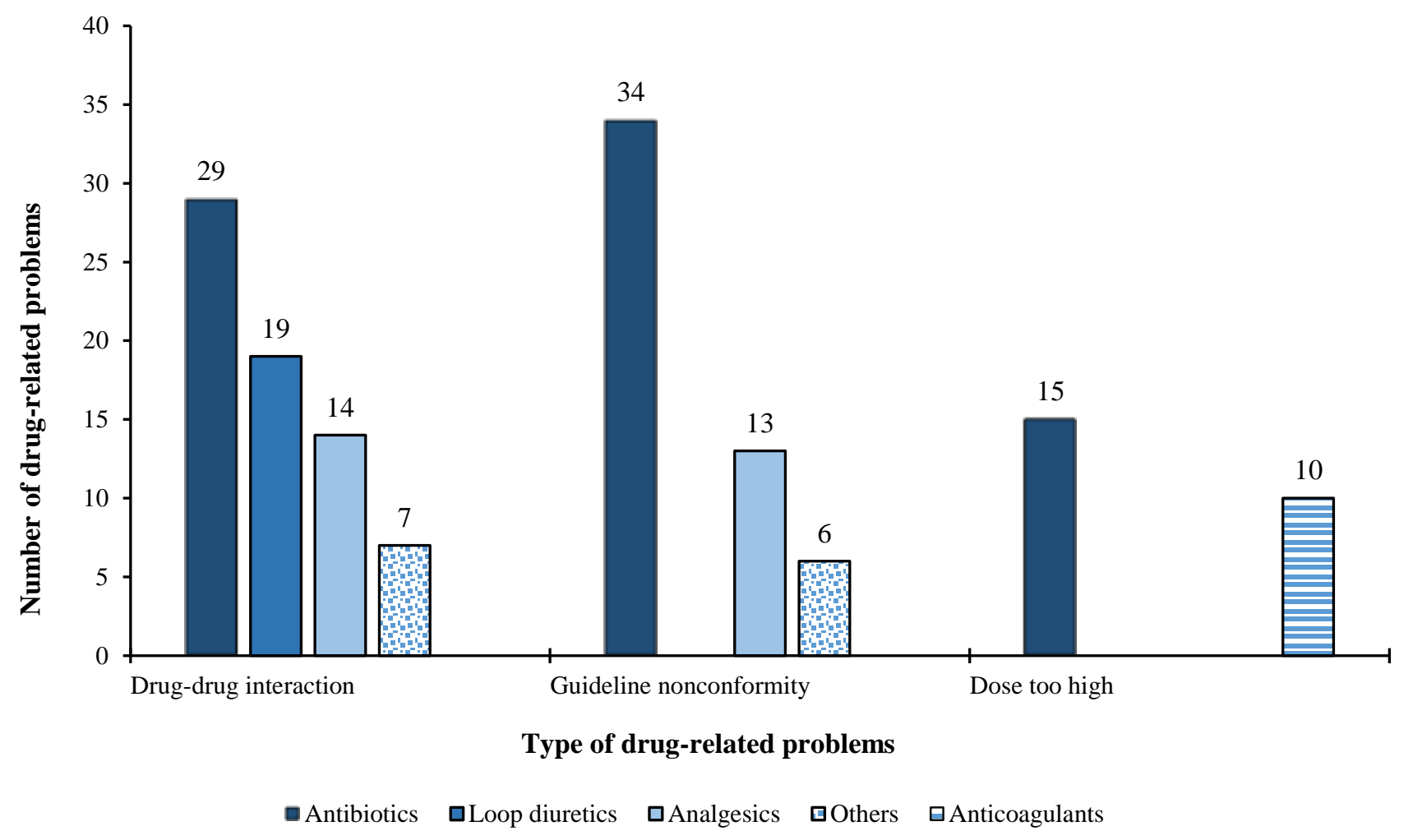

Figure 1: Most common medications involved in most common types of identified drugrelated problems.

CONCLUSION

The collaboration of clinical pharmacist with multidisciplinary MICU healthcare team is beneficial for identifying DRPs among critically ill patients and reporting these DRPs to MICU physicians. Most common types of identified DRPs indicate the need for educational interventions among healthcare providers.

\section{REFERENCES}

1. Rothschild JM1, Landrigan CP, Cronin JW, et al. The Critical Care Safety Study: The incidence and nature of adverse events and serious medical errors in intensive care. Critical Care Medicine. 2005; 33(8):1694-1700.

2. Latif A, Rawat N, Pustavoitau A, Pronovost PJ, Pham JC. National study on the distribution, causes, and consequences of voluntarily reported medication errors between the ICU and non-ICU settings. Critical Care Medicine. 2013 Feb; 41(2):38998.

3. Moyen E, Camire E, Stelfox Henry Thomas. Clinical review: Medication errors in critical care. Critical Care. 2008; 12(2):208. 
4. Eric Camiré, Eric Moyen, Henry Thomas Stelfox. Medication errors in critical care: risk factors, prevention and disclosure. CMAJ. 2009; 180(9): 936-941.

5. Cullen DJ, Sweitzer BJ, Bates DW, Burdick E, Edmondson A, Leape LL. Preventable adverse drug events in hospitalized patients: a comparative study of intensive care and general care units. Crit Care Med. 1997; 25(8):1289-97.

6. Donchin Y, Seagull FJ. The hostile environment of the intensive care unit. Curr Opin Crit Care. 2002; 8(4):316-20.

7. Kane-Gill Sandra, Weber Robert J. Principles and practices of medication safety in the ICU. Critical Care Clinics. 2006; 22(2):273-290.

8. Krähenbühl-Melcher A, Schlienger R, Lampert M, Haschke M, Drewe J, Krähenbühl S. Drug-related problems in hospitals: a review of the recent literature. Drug Saf. 2007; 30(5):379-407.

9. Claus BO, Robays H, Decruyenaere J, Annemans L. Expected net benefit of clinical pharmacy in intensive care medicine: a randomized interventional comparative trial with matched before-and-after groups. Journal of Evaluation in Clinical Practice. 2014 Dec; 20(6):1172-9.

10. Chisholm-Burns MA, Kim Lee J, Spivey CA, et al. United States pharmacists' effect as team members on patient care: systematic review and meta-analyses. Medical Care. 2010 Oct; 48(10):923-33.

11. Beckmann U, Gillies DM, Berenholtz SM, Wu AW, Pronovost P. Incidents relating to the intra-hospital transfer of critically ill patients. An analysis of the reports submitted to the Australian Incident Monitoring Study in Intensive Care. Intensive Care Med. 2004; 30(8):1579-85.

12. Escobar GJ, Greene JD, Gardner MN, Marelich GP, Quick B, Kipnis P. Intra-hospital transfers to a higher level of care: contribution to total hospital and intensive care unit (ICU) mortality and length of stay (LOS). J Hosp Med. 2011; 6(2):74-80.

13. Leape LL, Bates DW, Cullen DJ, et al. Systems analysis of adverse drug events. ADE Prevention Study Group. JAMA. 1995; 274(1):35-43.

14. Kopp BJ, Erstad BL, Allen ME, Theodorou AA, Priestley G. Medication errors and adverse drug events in an intensive care unit: direct observation approach for detection. Crit Care Med. 2006; 34(2):415-25.

15. Singh D, Agusti A, Anzueto A, et al. Global Strategy for the Diagnosis, Management, and Prevention of Chronic Obstructive Lung Disease: the GOLD science committee report 2019. Eur Respir J. 2019; 18(5):53. 
16. Rice KL, Leatherman JW, Duane PG, et al. Aminophylline for acute exacerbations of chronic obstructive pulmonary disease. A controlled trial. Ann Intern Med. 1987; 107(3):305-9.

17. Barr RG, Rowe BH, Camargo CA. Methylxanthines for exacerbations of chronic obstructive pulmonary disease. Cochrane Database Syst Rev. 2003; 89(2):2168.

18. Duffy N, Walker P, Diamantea F, Calverley PM, Davies L. Intravenous aminophylline in patients admitted to hospital with non-acidotic exacerbations of chronic obstructive pulmonary disease: a prospective randomised controlled trial. Thorax. 2005; 60(9):7137.

19. Leape LL, Cullen DJ, Clapp MD, et al. Pharmacist participation on physician rounds and adverse drug events in the intensive care unit. JAMA. 1999; 282(3):267-70.

20. Klopotowska JE, Kuiper R, van Kan HJ, et al. On-ward participation of a hospital pharmacist in a Dutch intensive care unit reduces prescribing errors and related patient harm: an intervention study. Crit Care. 2010; 14(5):R174.

21. Candice R. Preslaski, Ishaq Lat, Robert MacLaren, Jason Poston. Pharmacist Contributions as Members of the Multidisciplinary ICU Team. CHEST. 2013; 144(5):1687-1695.

22. Elisabeth TJ, Stine MH, Ann SM, Lars MY. Effects of implementing a clinical pharmacist service in a mixed Norwegian ICU. European Journal of Hospital Pharmacy. 2016; 23(48):197-202.

23. Behzad NA. Clinical Impact of Pharmacist Presence in ICU Medical Team on Mortality Rate. Clinicians. 2016; 1(4):15-33.

24. Nibal RC, Rony Z, Hanine M. Impact of clinical pharmacy interventions on medication error nodes. International Journal of Clinical Pharmacy. 2016; 38(6):1436-1444.

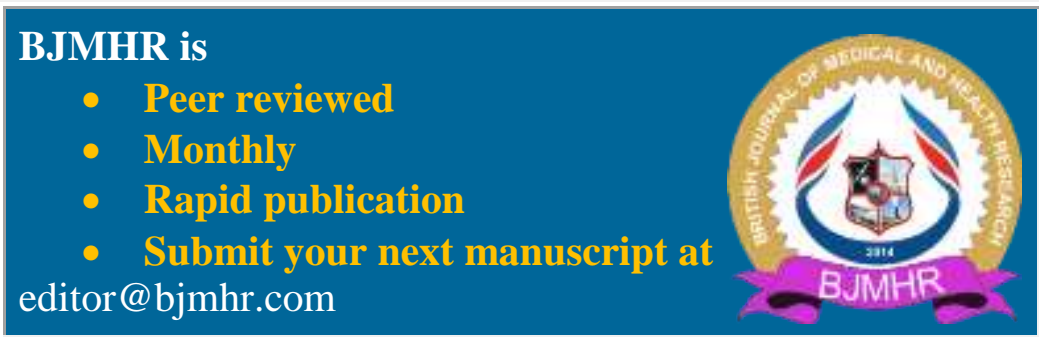

